\title{
ON ANOSOV DIFFEOMORPHISMS ON THE PLANE
}

\author{
P. MENDES
}

\begin{abstract}
A diffeomorphism on the plane holding an integrable Anosov splitting is called an Anosov diffeomorphism on the plane. In this paper the author proves that Anosov diffeomorphisms on the plane are structurally stable.
\end{abstract}

We start with some notations and definitions.

$R^{2}$ and $\operatorname{Diff}\left(R^{2}\right)$ stand, respectively, for the plane and its $C^{1}$-diffeomorphisms with the $C^{1}$-Whitney topology. Let $\operatorname{Per}(f)$ be the set of periodic points of $f$ and $\Omega(f)$ the set of its nonwandering points. If $x \in R^{2}, \alpha_{f}(x)$ and $\omega_{f}(x)$ denote the $\alpha$-limit set and the $\omega$-limit set of $x$.

$f, g \in \operatorname{Diff}\left(R^{2}\right)$ are conjugated if there is an homeomorphism $h: R^{2} \rightarrow R^{2}$ satisfying the equation $g h=h f$.

$f \in \operatorname{Diff}\left(R^{2}\right)$ is structurally stable if it is conjugated with every $g$ in some neighborhood of $f$.

An Anosov splitting for $f \in \operatorname{Diff}\left(R^{2}\right)$ consists of a complete Riemannian metric $\mu$ on $T R^{2}$, a continuous invariant splitting $T R^{2}=E^{s} \oplus E^{u}$, and a constant $\lambda \in(0,1)$, such that

$$
\begin{array}{ll}
\left\|D f^{n}(v)\right\| \leqslant \lambda^{n}\|v\|, & \text { for } v \in E^{s} \\
\left\|D f^{n}(v)\right\| \leqslant \lambda^{-n}\|v\|, & \text { for } v \in E^{u},
\end{array}
$$

where $E^{s}(x)$ and $E^{u}(x)$ has dimension 1 for all $x$, and \|\| is the norm induced by $\mu$. By the contractibility of the plane there are unit continuous vector fields $v^{s}$ and $v^{u}$, on $R^{2}$, generating $E^{s}$ and $E^{u}$, respectively. A diffeomorphism $f: R^{2} \rightarrow R^{2}$ admitting an Anosov splitting will be called an Anosov diffeomorphism if $v^{s}$ and $v^{u}$ have unique integral curves through each point of $R^{2}$, that is, if $E^{s}$ and $E^{u}$ are integrable line fields on the plane.

Let $F^{s}$ and $F^{u}$ be the arising continuous foliations. Set $L_{\infty}=L_{\infty}(f)=\{x$ $\in R^{2}: \alpha_{f}(x)=\phi$ or $\left.\omega_{f}(x)=\phi\right\}$. We recall that $\infty$ is $f$-strong attractor of $x \in L_{\infty}$ if there is a neighborhood $V_{x}$ of $x$ such that each compact set $K \subset R^{2}$ intersects only a finite number of the sets $f^{n}\left(V_{x}\right), n \geqslant 0$. Dually, we define $\infty$ is $f$-strong repeller of $x \in L_{\infty}$. Our result is the following

THEOREM 1. The Anosov diffeomorphisms of the plane are structurally stable.

We recall a structural stability theorem of [2].

Received by the editors April 19, 1976.

AMS (MOS) subject classifications (1970). Primary 34C35; Secondary 34D30.

Key words and phrases. Diffeomorphism, integrable Anosov splitting, structurally stable. 
THEOREM. Let $f \in \operatorname{Diff}\left(R^{2}\right)$ be such that

(1) $\Omega(f)$ is discrete;

(2) the periodic points of $f$ are hyperbolic;

(3) the invariant manifolds of its periodic points are in general position;

(4) $\infty$ is f-strong attractor or f-strong repeller of each point in $L_{\infty}$.

Then $f$ is structurally stable.

The proof of Theorem 1 is done using this theorem and the following propositions.

Proposition 1.1. If $f \in \operatorname{Diff}\left(R^{2}\right)$ has an Anosov splitting, $\Omega(f)=\operatorname{Per}(f)$.

Proposition 1.2. If $f \in \operatorname{Diff}\left(R^{2}\right)$ is Anosov, $\Omega(f)=\phi$ or $\Omega(f)=\{p\}$.

Proposition 1.3. If $f \in \operatorname{Diff}\left(R^{2}\right)$ is Anosov and $x \in L_{\infty}$ then $\infty$ is $f$-strong attractor or $f$-strong repeller of $x$.

Trivial examples of Anosov diffeomorphisms on the plane are the linear mappings with real eigenvalues $\lambda_{1}, \lambda_{2}$ such that $0<\left|\lambda_{1}\right|<1$ and $\left|\lambda_{2}\right|>1$. The translations are the only nontrivial examples up to the present, [5].

Conjecture. Each Anosov diffeomorphism on the plane is conjugate to one of the examples above.

Finally we state two problems.

Problem 1. Is the set of Anosov diffeomorphisms on the plane open in $\operatorname{Diff}\left(R^{2}\right)$ ?

Problem 2. If $f \in \operatorname{Diff}\left(R^{2}\right)$ has an Anosov decomposition is $f$ Anosov?

It is a pleasure to acknowledge numerous helpful conversations with $\mathrm{W}$. de Melo, J. Palis, C. C. Pugh and R. C. Robison. A substantial part of this work was done at Warwick University, during the 1974 Symposium on Dynamical Systems, and I would like to thank the members for their hospitality.

Proof of Proposition 1.1. Let $\alpha: \mathbf{R} \rightarrow \mathbf{R}^{2}$ be an orbit of $v^{s}$ and $t_{n} \in \mathbf{R}$ be a sequence such that $t_{n} \rightarrow \infty$ and $\alpha\left(t_{n}\right) \rightarrow p \in \mathbf{R}^{2}$. Then for some large $n$ there is an arc $l$ from $\alpha\left(t_{n}\right)$ to $\alpha\left(t_{n+1}\right)$ such that the closed piecewise differentiable curve $\Gamma=l \cup \alpha\left(\left[t_{n}, t_{n+1}\right]\right)$ is transversal to $v^{u}$. To see the existence of $l$ consider a small box

$$
B=\left\{p+x_{s} V^{s}(p)+x_{u} V^{u}(p), x_{s}, x_{u} \in(-\varepsilon, \varepsilon) \subset \mathbf{R}\right\}
$$

such that the angle between the vectors $V^{s}(x)$ and $V^{s}(p)$ is less than the one between the vectors $V^{u}(p)$ and $V^{s}(p)$, for all $x \in B$, and take $n$ such that $\alpha\left(t_{n}\right), \alpha\left(t_{n+1}\right) \in B$. The existence of $\Gamma$ contradicts the fact that $V^{u}$ is a continuous unit vector field. This argument shows that $\alpha$ is an embedding with closed unbounded image separating the plane. This is just the case for the stable manifold $W^{s}(p)$ of $p \in \operatorname{Per}(f)$. Setting $W^{s}=\cup_{p \in \operatorname{Per}(f)} W^{s}(p)$, from the unboundedness of each $W^{s}(p)$ it follows that the connected components $U_{i}, i=1,2, \ldots$ of $R^{2}-\bar{W}^{s}$ are simply connected open sets and thus diffeomorphic to $R^{2}$, by the Riemann mapping theorem, [1].

The set $R^{2}-\overline{W^{s}}$ is $f$-invariant and then, $f^{n}$-invariant for all $n \in Z$. For 
each $n, f^{n}$ sends connected components of $\mathbf{R}^{2}-\overline{W^{s}}$ onto connected components of $\mathbf{R}^{2}-\overline{W^{s}}$, that is, $f^{n}\left(U_{i}\right)=U_{j}$ for some $j$. Fix $U_{i}$. We have either, $f^{n_{i}}\left(U_{i}\right)=U_{i}$ for some $n_{i}$, or $f^{n}\left(U_{i}\right), n \in Z$, are disjoint open sets. In the first case the restriction $f_{\mid U_{i}}^{n_{i}}: U_{i} \cong R^{2} \hookleftarrow$ is a diffeomorphism of $R^{2}$ with no periodic points. Then, from [2], we have $\Omega\left(f_{\mid U_{i}}^{n}\right)=\phi$, which implies $\Omega(f) \cap U_{i}$ $=\phi$. In the second, of course we have also $\Omega(f) \cap U_{i}=\phi$. Thus $\Omega(f) \subset \overline{W^{s}}$. Similarly

$$
\Omega(f) \subset \overline{W^{u}}=\overline{\bigcup_{p \in \operatorname{Per}(f)} W^{u}(p)}
$$

and then $\Omega(f) \subset \overline{W^{s}} \cap \overline{W^{u}}$. Using the linear independence of $v^{s}$ and $v^{u}$ and the $\lambda$-Lemma [3], we get $\overline{W^{s}} \cap \overline{W^{u}}=\operatorname{Per}(f)$, which ends the proof.

Proof of Proposition 1.2. We note that (1.2) being true for some power of $f$ it will be true for $f$ itself. Then we have only to prove the following:

$$
\begin{aligned}
& \text { If } f \in \operatorname{Diff}\left(R^{2}\right) \text { is Anosov and } p \in \Omega(f) \text { is a fixed point } \\
& \text { then } \Omega(f)=\{p\} \text {. }
\end{aligned}
$$

To prove (*) we will use some common expressions as "on the right", "on the left", "above", "below", etc., with the obvious technical meaning in each case. Let $x_{1}, x_{2} \in W^{s}(p), x_{1}$ on the right of $p$ and $x_{2}$ on its left, and $y_{1}, y_{2} \in$ $W^{u}(p), y_{1}$ above $p$, and $y_{2}$ below. Let $\alpha, \beta$ be the orbits of $v^{u}$ through $x_{1}, x_{2}$, and $\gamma, \delta$ the orbits of $v^{s}$ through $y_{1}, y_{2}$, respectively. If $x_{i}, y_{i}, i=1,2$, are sufficiently near $p, \alpha, \beta, \gamma, \delta$ bound a square $Q$ containing $p$ with the following property: if $q \in \operatorname{Per}(f) \cap Q, q \neq p$, then the orbit of $q$ has points outside $Q$.

Then it is enough to prove that $f$ has no periodic points outside $Q,(1)$ on the right of $\alpha$; (2) on the left of $\beta$; (3) above $\gamma$; and (4) below $\delta$. Now we proceed to prove (1).

Let $B$ be the band bounded by $\alpha$ and $f(\alpha)$. It is clear that $f^{n}(B)$ is the band bounded by $f^{n}(\alpha)$ and $f^{n+1}(\alpha), n \in Z$, and then int $f^{n}(B) \cap$ int $f^{m}(B)=\phi$ if $n \neq m$. Thus $\operatorname{Per}(f) \cap\left(\cup_{n \in Z} f^{n}(B)\right)=\phi$. We note that $\cup_{n \in Z} f^{n}(B)$ is open. Moreover,

$$
\begin{aligned}
& \text { if } x \in R^{2} \text { is on the right of } \alpha \text { and on the left of } f^{-n}(\alpha) \text {, } \\
& n>0 \text {, then } x \in \cup_{n>0} f^{-n}(B) .
\end{aligned}
$$

Let $P$ be the open region on the right of $\alpha$. Set $A^{-}=\cup_{n \in Z} f^{n}(B) \cap P$, $A^{+}=P-\bar{A}$ and $F=\sigma A^{-}=\sigma A^{+}(\sigma=$ topological boundary $)$. From what was stated above we get $f(F)=F$ and $f\left(A^{+}\right)=A^{+}$. Consider a connected component $C$ of $A^{+}, x \in \sigma C$ and $\alpha_{x}$ the orbit of $v^{u}$ through $x$. The orbit $\alpha_{x}$ separates $P$ in two open regions $P^{+}$and $P^{-}$, with $P^{+}$on the right of $\alpha_{x}$. Using (**) we get by contradiction that $P^{+} \cap \overline{A^{-}}=\phi$. Then $P^{+} \subset C$. This 
and the local triviality of the continuous foliation $F^{u}$, assure that $P^{+}=C$, and that $\alpha_{x}$ is a connected component of $F$.

If $\operatorname{Per}(f) \cap A^{+} \neq \phi, f^{n}(C)=C$ for some connected component of $A^{+}$ and some $n>0$, just because $f\left(A^{+}\right)=A^{+}$. Thus $f^{n}\left(\alpha_{x}\right)=\alpha_{x}$ for the connected component $\alpha_{x}=\sigma C$ of $F$. $\alpha_{x}$ being an orbit of $v^{u},\left.f^{-n}\right|_{\alpha_{x}}: \alpha_{x} \rightarrow \alpha_{x}$ is a contraction and then has a fixed point $q$.

It is easy to check that $f^{-m}\left(\alpha_{x}\right) \cap W_{f^{n}}^{s}(q)=\{y\}$. We note that $q$ is on the right of $f^{-m}(\alpha)$, and then $f^{n}(y)$ is also on the right of $f^{-m}(\alpha)$. Thus the same occurs with $f^{n}\left(f^{-m}(\alpha)\right)$ which is a contradiction. This proves that $\operatorname{Per}(f) \cap$ $A^{+}=\phi$. In the same way we conclude that $\operatorname{Per}(f) \cap F=\phi$, which ends the proof of (1). The proofs of (2), (3) and (4) are similar, and (1.2) is proved.

Up to now we have been analysing the nonwandering set of an Anosov diffeomorphism concluding that it is very nice: it is empty or consists of a single point. Now we analyse the set $L_{\infty}$.

Proof of Proposition 1.3. Take a neighborhood $Q$ of $x$ trivial for the continuous foliations $F^{s}$ and $F^{u}$, such that $f^{n}(Q) \cap Q=\phi$ for all $n \neq 0$. Let $\beta^{s}=\gamma_{x}^{s} \cap Q$ and $\beta^{u}=\gamma_{x}^{u} \cap Q$, where $x \in \gamma_{x}^{s} \in F^{s}$ and $x \in \gamma_{x}^{u} \in F^{u}$. They can be supposed connected by taking $Q$ sufficiently small. If $\infty$ is not $f$-strong attractor of $x$ there are points $y_{i} \in Q, i=1,2, \ldots$, and integers $0<n_{1}<n_{2}<\ldots$ such that $f^{n_{i}}\left(y_{i}\right) \rightarrow y$, for some $y \in R^{2}$.

Consider the points $\tilde{y}_{i}=\beta^{u} \cap \gamma_{y}^{s}$ and the arcs $\alpha_{i} \subset \gamma_{y_{i}}^{s}$, from $y_{i}$ to $\tilde{y}_{i}, i=1$, $2, \ldots$ Let $l\left(\alpha_{i}\right)$ be the length of $\alpha_{i}$. It is easy to see that there is $a=$ $\sup _{i} l\left(\alpha_{i}\right)$, and that $l\left(f^{n_{i}}\left(\alpha_{i}\right)\right) \leqslant a \lambda^{n_{i}}$. Thus $d\left(f^{n_{i}}\left(y_{i}\right), f^{n_{i}}\left(\tilde{y}_{i}\right)\right) \rightarrow 0$ and then $f^{n_{1}}\left(\tilde{y}_{i}\right) \rightarrow y$. From this we get $i_{0}$ such that $f^{n_{i}}\left(\beta^{u}\right) \cap \gamma_{y}^{s} \neq \phi$ for all $i \geqslant i_{0}$, by taking a neighborhood of $y$ trivial for both foliations, $F^{s}$ and $F^{u}$. Setting $f^{-n_{10+1}}\left(\gamma_{y}^{s}\right)=\eta$ and $r=n_{i_{0}+1}-n_{i_{0}}$ we have $\eta \cap \beta^{u} \neq \phi$ and $f^{r}(\eta) \cap \beta^{u} \neq \phi$.

It is clear from the definition that if $\infty$ is $f^{r}$-strong repeller of $x, \infty$ is also $f$-strong repeller of $x$. Supposing, by contradiction that $\infty$ is not $f$-strong repeller of $x$, and thus, it is not $f^{r}$-strong repeller of $x$, we get by the arguments above an orbit $\xi$ of $v^{u}$ and an integer $n>0$, such that $\xi \cap \beta^{s} \neq \phi$ and $\left(f^{r}\right)^{n}(\xi) \cap \beta^{s} \neq \phi$. It is enough to prove that $f^{r}(\xi) \cap \beta^{s} \neq \phi$ because this implies that $f^{r}(Q) \cap Q \neq \phi$, which is a contradiction.

This will be proved by contradiction again. If $f^{r}(\xi) \cap \beta^{s}=\phi$ we have $f^{r}(\xi) \cap \xi=\phi$. Noting that $\xi$ separates $R^{2}$ in two open regions, let $P$ be the closure of the one containing $f^{r}(\xi)$. We can suppose without loss of generality that $f^{r}$ is orientation preserving. Thus

(a) $f^{r}(P) \subset$ int $P$,

(b) $f^{r}(P) \cap \beta^{s}=\phi$.

From (a) it follows by induction that $f^{n r}(P) \subset$ int $f^{r}(P)$. Then from (b) we get $f^{n r}(\xi) \cap \beta^{s}=\phi$ which is a contradiction.

\section{REFERENCES}

1. N. Levinson and R. M. Redheffer, Complex variables, Holden-Day, San Francisco, Calif., 1970. MR 42 \#6193. 
2. P. Mendes, On stability of dynamical systems on open manifolds, J. Differential Equations 16 (1974), 144-167. MR 49 \#9876.

3. J. Palis, On Morse-Smale dynamical systems, Topology 8 (1968), 385-404. MR 39 \#620.

4. J. Palis and S. Smale, Structural stability theorems, Proc. Sympos. Pure Math., vol. 14, Amer. Math. Soc., Providence, R.I. 1970, pp. 223-231. MR 42 \#2505.

5. W. White, An Anosov translation, Dynamical Systems (Proc. Sympos., Univ. of Bahia, Salvador, 1971), Academic Press, New York, 1973, pp. 667-670. MR 50 \#8605.

Departamento de Matemática, Universidade Federal de Minas Gerais, Instituto de Ciências Exatas, 30.000, Belo Horizonte, M.G., Brasil 